In Professor Davis's second paper it is pointed out that the 48 operations which bring the cube into coincidence with itself are operations which bring any one of the eight triangles into which a face is divided by its lines of symmetry into coincidence with the 48 like triangles of the surface of the cube. Any of the subgroups of operations brings this same triangle into coincidence with merely a part of the 48 triangles. A cube with these triangles all of one color and the rest of the cube another color is an object which, taking account of color, comes into coincidence with itself only under the operations of the subgroup. Similar methods can be used for the representation of all regular groups and their subgroups.

Thomas F. Holgate, Secretary of the Section.

\title{
ON THE HISTORY OF THE EXTENSIONS OF THE CALCULUS.
}

BY J. G. HAGEN, S. J.

THE results attained by means of the infinitesimal calculus naturally evoked similar attempts in other directions. We may distinguish between two kinds of new theories, differing from each other mainly in their origin. The one is the natural outcome of an ever recurring need of solving practical problems or of giving existing theories a broader basis; the other owes its origin to purely abstract speculation. The former is not the property of any one mathematician; its nomenclature is not arbitrarily chosen, and its general introduction is only a question of time. This is clear from the history of all those branches of mathematics that have come into general use.

The case is somewhat different with the purely speculative theories. But though these may never succeed in demonstrating their practical utility, yet they are of value to mathematical science since they throw new light on existing methods and on their usefulness. For our purpose, however, a summary outline of these theories will suffice.

The present abstract is confined to those theories that are in close relation to the infinitesimal calculus and the theory of functions, and excludes, for instance, all geometrical methods and what are called "principles" or methods of demonstration. 
In enumerating the following theories it cannot be the intention of the writer to classify them with the abstract theories that will never command general attention. 'They are mentioned merely because they have not yet been generally adopted. How long a new theory may remain a hidden treasure is amply illustrated by Grassmann's Ausdehnungslehre. Perhaps the reason why some of the theories considered have appeared of slight value is that they are formal in their nature and were invented before the full import and difficulty of Taylor's theorem was revealed. We expressly exclude from this remark Cauchy's calculus of residuals and the calculus of finite differences. How far the "operations" of this latter calculus may be promoted by the modern theory of functions is a question of great. interest, which has probably not yet been fully answered.

While some of these new theories that have a set of formulas similar to the infinitesimal calculus are generally known, a synoptical view of all of them may be instructive. The references to the original sources would be be very numerous and cumbersome and are omitted here, as they will be given elsewhere.*

\section{Derivation with a General Index.}

The attempts to invent a calculus, of which the differential and integral calculuses should be particular branches, date back as far as Leibniz. Their history was outlined by Liouville, Tardy, and Cantor. That a negative index of differentiation is equivalent to an integration, and vice versa, was first stated by Leibniz. The first examples, in which the index $n$ of an $n^{\text {th }}$ differential coefficient is put equal to $\frac{1}{2}$, were given by Leibniz and Euler. Later, Fourier expressed a differential coefficient by a double integral, and added the passing remark that the index of the differential may be any number. While these few examples were intended as mere curiosities, the theory of the new calculus was established by Licuville and has since remained substantially in the shape that he gave it, although it was perfected later by Riemann, Gruenwald, Most, Letnikoff, and Krug.

Liouville gave three independent definitions of the derivation with a general index: first by development into a series, then by finite differences, and finally by definite integrals.

1. Liouville supposed the given function to be developed into an exponential series, of which he took the $n^{\text {th }}$ differen-

\footnotetext{
* The writer's Synopsis of higher mathematics, vol. III., pp. 85-99.
} 
tial, and then assumed the index $n$ to be any number. His hypothesis is generally considered unsatisfactory. Riemann, while yet a student, chose for the same purpose a series progressing according to powers of the increment $h$ in the development of $f(x+h)$, such that the exponents increase by integral numbers. The $n^{\text {th }}$ derivation is then the coefficient of $k_{n} h^{n}$, where the factors $k_{n}$ are subject to the condition, that the development becomes Taylor's theorem when $n$ is an integer.

The definition by series has been abandoned by later writers on the subject.

2. A second definition was given by Liouville in the following manner: He expressed the finite difference $\Delta^{n} f$ by Newton's formula, then divided it by $h^{n}$, and finally took the limit for $h=0$. If then the index $n$ is taken arbitrarily, we have a definition for the general derivation. Gruenwald was probably the first to assume this index to be a complex number. He also expressly added a lower and upper limit to this derivation, as is required in a calculus which is to comprise the integral calculus as a special branch, and which may be valid only within definite limits in the plane of complex coördinates. Gruenwald's notation of the $n^{\text {th }}$ derivation of $f(x)$ within the interval from $a$ to $x$ seems to be the most convenient : $D^{n}[f(x)]_{a}^{x}$.

3. A third definition is that by definite integrals. Liouville gave two integrals with the limits zero and infinity, and all later definitions are only generalizations of these. Gruenwald, as before mentioned, changed the limits into $\alpha$ and $x$. Most gave four different integrals, in each of which the upper limit is a critical point of the function $f(x)$. Letnikoff's two integrals resemble those of Liouville, except for the upper limit $x$. Krug's treatise on the same subject seems to surpass all others by a more precise statement of the conditions to which the formulas are subject. One of his integrals is a curve integral, and reminds us of Cauchy's fundamental theorem; the other has the limits $a$ and $z$, and comprises all the cases discussed by Gruenwald and Most.

It will not be necessary for the purpose of this abstract to mention the various theorems which Liouville has established for the operation $D^{n}[f(x)]_{a}^{x}$.

\section{Cauchy's "Calcul des Résidues."}

This calculus is entirely due to Cauchy as to its origin and complete development. Unfortunately this calculus in 
its entirety has not yet gained general recognition. Bertrand's "Calcul différentiel" is probably the only textbook that gives a complete theory of it. Of course, as far as this calculus is connected with Cauchy's fundamental theorem of integration around contours, it will ever remain a corner stone in the theory of functions.

1. The "Calcul des résidues" is not a generalization of the infinitesimal calculus, like the derivation with general index, but rather a branch parallel to it. Cauchy was probably led to this new idea by Lagrange's definition of the derivative as coefficient of the increment $e^{+1}$ in the development of the function $f(z+e)$. For he defined the residuum as the coefficient of $e^{-1}$. This applies of course only to functions which have points of infinity. Suppose the equation $f(z)=\infty$ has the root $z_{r} m$ times; then the function $\left(z-z_{r}\right)^{m} f(z)=\varphi(z)$ is in the point $z=z_{r}$ neither zero nor infinite. Hence, putting $z=z_{r}+e$, we have the development

$$
\begin{array}{cr}
f\left(z_{r}+e\right)=\frac{\varphi\left(z_{r}+e\right)}{e^{m}}=\frac{1}{e^{m}} \varphi\left(z_{r}\right)+\cdots+\frac{1}{e} \frac{\varphi^{(m-1)}\left(z_{r}\right)}{1.2 \cdots(m-1)} \\
+\frac{\varphi^{(m)}\left(z_{r}+\theta e\right)}{1.2}, & \quad(\theta< \pm 1) .
\end{array}
$$

Here the coefficient of $\frac{1}{e}$ is called the residuum of $f(z)$ in regard to the $m$-fold $\operatorname{root} z_{r}$ :

$$
\frac{\varphi^{(m-1)}\left(z_{r}\right)}{(m-1) !} \equiv \frac{1}{(m-1) !} \frac{d^{m-1}}{d e^{m-1}}\left[e^{m} f\left(z_{r}+e\right)^{\dot{1}}\right]_{e=0} .
$$

2. The "integral residuum" of the same function $f(z)$ is defined as the sum of the residuals in regard to all the roots of $f(z)=\infty$ within certain limits, $i$. e., within $z=a+i A$ and $z=b+i B$, and is designated by

$$
{ }_{a}^{b} E_{A}^{B}((f)) \text { or }{ }_{a}^{b} E_{A}^{B}[f],
$$

where the letter $E$ is the initial of the word "Extraction." It was changed by Cauchy into a character somewhat similar to a capital epsilon.

The "partial residuum" refers to a function composed of factors $f_{1}, f_{2}, \cdots$, and is designated by enclosing in brackets that factor of which the residuum is taken. Thus, $E\left[f_{1}\right] f_{2} \cdots$ refers to the roots of $f_{1}=\infty$ alone. 
The sign $E$ follows all the rules of the sign of integration, so far as the associative, the distributive, and the commutative laws are concerned.

Cauchy has established many theorems of this calculus which it is not necessary to mention here. By numerous applications to nearly all branches of mathematics and physics he has proved the great power of his calculus.

\section{Schell's "Quotial and Instaural."}

This interesting but little known calculus is similar to Cauchy's "Calcul des résidues," in as much as it branches off from the definition of the differential in a new direction. But while Cauchy started from Lagrange's idea of the derivative, Schell takes Leibniz's and Euler's point of view. His original idea is to replace the limit of a finite difference by the limit of a finite quotient. $\mathrm{He}$ endeavored to establish a kind of dualism in the infinitesimal calculus by interchanging differences and quotients, sums and products, somewhat similar to Gergonne's and Poncelet's dualism in geometry, where line and point coördinates are interchanged.

There are only two other publications relating to this subject; the one by Walton on the symbolic operator $\left(x \frac{d}{d x}\right)^{r}$, the other by Gaussin on the "Definition du calcul quotientiel." Gaussin considers this calculus mainly from the arithmetical and algebraic point of view.

1. The definition of the "quotial" consists in this : The finite difference $\Delta x=x_{1}-x$ and the differential $\left.\lim _{x_{1}=x}\right\rfloor x=d x$ are replaced by the quotient $\theta x=x_{1}: x$ and the " quotial " $\lim _{x_{1}=x} \theta x=\vartheta x$ (or $=q x$ in Gaussin's designation). Hence $x_{1}=x+\Delta x$ has the dualistic formula $x_{1}=x \Theta x$. For the "quotial" of a function we have

$$
u=F(x), u_{1}=F(x \Theta x), \vartheta u=\lim _{u_{1}=u} \frac{u_{1}}{u}=\frac{F(x \vartheta x)}{F(x)} .
$$

Now, while in the differential calculus we pass from a differential to a differential quotient, we here pass from a "quotial" to a difference, by means of the logarithm. The peculiarity of this logarithm lies in the variable base $\theta x$, with the limit $\vartheta x=\omega=1$. It is denoted by $\lambda$, and we have the following definition :

Quotial Logarithm of $F(x)=\lambda \vartheta F(x)=\lambda \frac{F(x \vartheta x)}{F(x)}$. 
The connection between this and the differential calculus appears from the following transformation :

$$
\lambda \vartheta F(x)=\frac{x}{F(x)} \frac{d F(x)}{d x}=x \frac{d}{d x} \lg F(x) .
$$

which shows that the "quotial derivative" is substantially identical with a logarithmic differentiation.

On the other hand, it appears that "quotial derivatives" of higher order with the symbol

$$
\lambda \vartheta^{r} \equiv\left(x \frac{d}{d x} \lg \right)^{r}
$$

are not quite identical with Walton's operator $\left(x \frac{d}{d x}\right)^{r}$.

2. The "instaural," as the limit of a product, is the dualistic counterpart of the integral, the limit of a sum. We first write the quotial derivative in this form

$$
\lambda \vartheta F(x)=\lambda \frac{F(x \omega)}{F(x)}=f(x) \text {, or } \frac{F(x \omega)}{F(x)}=\omega^{f(x)} \quad(\lim \omega=1) .
$$

Then we divide the quotient of two given limits $x_{0}$ and $x$ into $n$ arbitrary factors

$$
\frac{x}{x_{0}}=\omega_{1} \cdots \omega_{n},
$$

and substitute the $n$ values

$$
x_{0}, \quad x_{1}=x_{0} \omega_{1}, \quad x_{2}=x_{1} \omega_{2}, \quad \cdots, \quad x_{n-1}=x_{n-2} \omega_{n-1},
$$

in the above formula (putting at the same time for $\omega$ successively $\left.\omega_{1}, \cdots, \omega_{n}\right)$. Finally, multiplying all these equations, and remembering that $\omega_{r+1}=\frac{x_{r+1}}{x_{r}}=\vartheta x_{r}$, we have the product

$$
\frac{F(x)}{F\left(x_{0}\right)}=\omega_{1}^{f\left(x_{0}\right)} \cdots \omega_{n}^{f\left(x_{n-1}\right)}=P \omega_{r+1}^{f\left(x_{r}\right)}=P \vartheta x_{r}^{f\left(x_{r}\right)} .
$$

If this product has a definite finite limit, it is called the "instaural of $f(x)$ between $x_{0}$ and $x$ ("produit" according to Gaussin), and designated by

$$
\frac{F(x)}{F\left(x_{0}\right)}={\stackrel{x}{P} \vartheta{ }^{\prime}}_{x_{0}}^{f(\sigma)}, \quad \text { or } \quad P \vartheta \sigma^{f(\sigma)}=\text { const. } F(x) .
$$


The connection between this formula and the integral calculus appears from the following identities:

$$
\begin{gathered}
f(x)=x \frac{d}{d x} \lg F(x), \text { or } C \cdot \exp \int \frac{f(x)}{x} d x=F(x), \\
\stackrel{x}{P} \vartheta_{x_{0}} f(\sigma)=e^{\int_{x_{0}}^{x} \frac{f(\sigma)}{\sigma} d \sigma}
\end{gathered}
$$

IV. The Exponential Function of Higher Order.

In considering the product as a repeated sum, and the power as a repeated product, it was but natural to look for a new expression which would repeat the power, and thus increase the number of elementary operations from seven to eight. The attempt, however, failed, for the reason, that base and exponent are not commutative, as are terms: and factors. Yet we meet in the older literature the expression $r^{.^{.}}$. It is not known where it occurs first. Condorcet equated this power of higher order to a certain series, without giving any proof. Euler, in supplying this proof, gave a full discussion of the function, and found an interesting relation between it and the base of natural logarithms.

1. He puts $\beta=r^{a}, \gamma=r^{\beta}, \delta=r^{\gamma}, \cdots$ and investigates the condition that the series $\alpha, \beta, \gamma, \cdots$ converge toward a finite value $\omega$. Evidently this condition is $r^{\omega}=\omega$.

Euler then finds that the maximum value of the root $r$ which can satisfy this condition is $r_{1}=e^{1 / e}=1.44 \cdots$ and the corresponding value $\omega=e=2.7 \cdots$ For all values $r>r_{1}$ the limit $\omega=x+i y$ is imaginary, and for all values $r<r_{1}$ the condition $r^{\omega}=\omega$ furnishes generally two values $\omega$ and $\omega^{\prime}$, towards which the series $\alpha, \beta, \gamma, \cdots$ can converge.

2 . Assuming the root $r=e$ and writing $x$ for $a$ the function may be expressed by the symbol $e^{e^{e^{\cdot x}}}=\exp ^{n} x$, where "exp" is Cayley's notation for the exponential, and where $n$ indicates the number of times the letter $e$ is written. In this case the differential of the function can be written in the following manner :

$$
\frac{d}{d x} \exp ^{n} x=\exp ^{n} x \cdot \exp ^{n-1} x \cdots \exp x .
$$

3. There are later essays on this function by Eisenstein and Woepke, which give no reference to Euler's treatise. They do not seem to have substantially advanced our knowledge of the nature of this function. 


\section{Extension of the Calculus of Finite Differences.}

There are two very interesting new theories in the line of finite differences: one by Oettinger and the other by Dr. McClintock.

1. Oettinger creates a calculus perfectly analogous to the calculus of finite differences, another imitation of the geometric dualism. He runs side by side the two formulas

$$
\Delta f(x)=f(x+h)-f(x) \text { and } \quad \xi f(x)=f(x+h)+f(x) \text {, }
$$

and all their consecutive corollaries. The original idea is the change of the minus in the former formula into plus in the latter.

The letter $\zeta$ (instead of $\Delta$ ) is the initial of the word "Zunahme" (increase). Oettinger then develops a full theory of $\zeta^{ \pm m}$ parallel to that of $\Delta^{ \pm m}$, with many formulas and applications. This calculus does not seem to occur in the later literature.

2. Dr. McClintock has laid down his new calculus in an elaborate essay, which appeared in an early volume of the American Journal of Mathematics. He starts from the following symbol, where $D$ is used for $\frac{d}{d x}$ as usual,

$$
E^{h} \varphi(x) \equiv e^{h D} \varphi(x) \equiv(1+\Delta) \varphi(x) \equiv \varphi(x+h), \Delta x=h,
$$

and designates the operation " $E$ " as "enlargement."

A number of symbolic formulas with the operator $E$ is then established, of which the following is remarkable for its simplicity, and seems to be the most important of all :

$$
D \equiv \lg E .
$$

Dr. McClintock then develops the theory of the functions of $E$, and uses it to give the theory of differentiation a broader basis, as the logarithmic branch of the doctrine of these functions.

It would be too long in this abstract to show how the formulas of the differential calculus are derived from the symbol $D=\lg E$. Nor can we do more than mention the remarkable application of this new method for finding simultaneously all the roots of an equation. It is hoped that this calculus of enlargement will give a new impulse to the use of symbolic operations which have proved so powerful in the hands of French and English mathematicians. 


\section{The Logarithmic Methods of Bergbohm and Oltramare.}

The facility with which exponentials can be handled in differentiating and integrating seems to have given rise to the two methods to be mentioned here. Both have this in common, that they pass from a given function to a logarithm or exponential, and, after a series of suitable transformations, back to the given function. This process appears almost like a roundabout way, by which the direct differentiation and integration is partly covered. Both methods were represented by their authors in a rather obscure and unsatisfactory manner.

1. The "new methods" of Bergbohm are based upon the symbol $d x^{d y}$ with vanishing base and exponent. This symbol is first developed into the converging series

$$
d x^{d y}=e^{d y \lg d x}=1+\frac{d y \lg d x}{1}+\frac{(d y \lg d x)^{2}}{1.2}+\cdots,
$$

from which the fundamental formula is obtained

$$
d x^{d y}=1+d y \lg d x .
$$

Of this formula the logarithm is taken, and then the antilogarithm or numerus, both in regard to a certain vanishing base $d \beta$. These operations are designated by $\lambda$ and $\nu$. In this manner the further formulas are derived

$$
\frac{d y \lg d x}{\lg d \beta}=\lambda(1+d y \lg d x), \nu \frac{d y \lg d x}{\lg d \beta}=1+d y \lg d x,
$$

since $\lambda d x . \lg d \beta=\lg d x$.

The application consists in this, that by the above mentioned transformations any expression $d f$ is changed into $f^{\prime} d x$, and again any expression $f^{\prime} d x$ into $d f$. By the former process the derivative is obtained, by the latter the integral. The method is tested by some of the elementary formulas.

2. Oltramare's "Calcul de generalisation" rests on the symbol

$$
G e^{x u+y v+z w+\cdots}=\varphi(x, y, z, \cdots),
$$

by which the operation with the "characteristic" $G$ is said to be defined.

The partial generalization, which refers to $u$ only, or to $v$ only, etc., is denoted by $G_{u}, G_{v}, \cdots$; and a repeated generalization by $G^{2}, G^{3}$, etc. The inverse operation $G^{-1}$ is called "degeneralisation." 
The principal operations in this calculus consist in developing both members of the equation

$$
G e^{(x+a) u+(y+b) v+\cdots}=\varphi(x+a, y+b, \cdots)
$$

according to powers of the increment $a$, and equating the coefficients of equal powers. By this process the following fundamental formula is found :

$$
G e^{x u+y v+\cdots} \psi(u, v, \cdots)=\psi\left(D_{x}, D_{y}, \cdots\right) \varphi(x, y, \cdots) .
$$

In the applications the function $\psi$ has to be developed according to powers of $D_{x}=\frac{d}{d x}, D_{y}=\frac{d}{d y}, \ldots$, after which the differentiations are to be performed.

The idea of this calculus seems to be to replace a given function $\varphi(x, y, \cdots)$ by the exponential function $G e^{x u+\cdots}$, and then, after some suitable transformations, to return from the exponential to the given function. This suggests the idea that $G$ is an operator like $\Sigma$, although the author avoids saying so, perhaps owing to the objections which had been raised against Liouville's assumption, that every function can be developed into an exponential series. The work of Oltramare was recently reviewed by Professor Lovett in the BuLletin.

GEORG ETOWN UNIVERSITY,

May, 1900.

\section{BURNSIDE'S THEORY OF GROUPS.}

Theory of Groups of a Finite Order. By W. Burnside,* M.A., F.R.S.; Professor of Mathematics at the Royal Naval Colr lege, Greenwich. Cambridge, The University Press, 1897. 8vo., xvi +388 pp.

THIs work enjoys the distinction of being the first treatise on the theory of groups which does not consider the applica-

*The joint author of Burnside and Panton's "Theory of Equations" is W. S. Burnside, an Irishman, professor of mathematics and fellow of Trinity College, Dublin. $\mathrm{He}$ is not related to the author of the work here considered, who is of Scotch extraction and has not published any other book. He has, however, published a number of memoirs-most of which relate to the theory of groups-in the Proceedings of the London Mathematical Society and in the Messenger of Mathematics. It seemed desirable to mention these facts since the two authors-William Burnside and William Snow Burnside-are frequently confused. Cf. Netto's review, Schlömilch's Zeitschrift, vol. 44 (1899), p. 20. 